SPhT t96/148

December 1996

\title{
A Note on the QCD Vacuum Tensor Susceptibility.
}

\author{
V.M. Belyaev* \\ SPhT, CEA-SACLAY, 91191 Gif-sur-Yvette, CEDEX, France \\ A. Oganesian \\ ITEP, 117259, Moscow, Russia
}

\begin{abstract}
We consider the procedure of determination of induced quark condensates in the QCD sum rule approach. It is noted that the previous estimations of this value were very rough. It is shown, that the detailed analyses of this parameter of QCD vacuum leads to the conclusion that the value of the tensor susceptibility has opposite sign and much larger than estimations which were obtained before. The reason of this contradiction is discussed. It is noted that naive ways of determination of induced condensates may lead to wrong results. The case of tensor susceptibility is the example demonstrating the importance of the procedure which is applied for calculation of such condensates. New results for the nucleon tensor charge are presented. The tensor charge is related to the first moment of the transversity distribution function $h_{1}(x)$.
\end{abstract}

PACS number(s): 11.15.Tk, 11.55.Hx, 12.38.Lg, 13.60.Hb

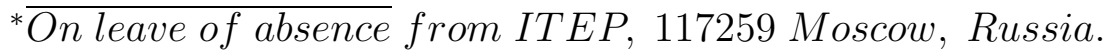




\section{Introduction}

The QCD sum rule approach suggested by Shifman, Vainshtein and Zakharov [[1] is a useful tool to study various properties of hadrons (see, for example [2]). Ioffe suggested to use this approach to study properties of baryons [3]. This method has been applied for calculations of magnetic moments [4, 5] and axial charge [6] of baryons. In order to determine static properties of hadrons it was suggested to consider correlators of quark currents in the presence of external constant classical field where nonperturbative effects are taken into account in the so-called induced condensates [4] or (which is the same) in bilocal operators [5]. This approach was extended to the case of variable external field [7].

Recently, this version of QCD sum rules has been used for the evaluation of nucleon tensor charge [8, 9]. The value of nucleon tensor charge is related to the first moment of the transversity distribution function $h_{1}(x)$ [10], where $h_{1}(x)$ is an additional twist-two chirality violating structure function which can be measured in the Drell-Yan process with both beam and target transversely polarized [11].

To make a reasonable estimation for nucleon tensor charge, it is necessary to evaluate nonperturbative effects which are related to induced condensates. The so-called tensor susceptibility corresponds to the lowest dimension nonvanishing operator and may play an important role in QCD sum rule analyse. The first estimations for this tensor susceptibility were made in [8], where the authors claimed that this value is small and does not effect on their result for nucleon tensor charge.

Here, we present new calculations of the tensor susceptibility and reanalyse the QCD sum rules for tensor charge obtained in [8, 9]. It is shown that this susceptibility is several times larger and has opposite sign than the first estimation.

The first results for the structure function $h_{1}(x)$ in QCD sum rule approach has been made by Ioffe and Khodjamirian [12]. They used QCD sum rules for four-point correlators which were in [13] to study structure functions of deep-inelastic scattering. 


\section{Tensor Susceptibility}

Let us consider the correlator of two tensor currents:

$$
\begin{array}{r}
\int d^{4} x e^{i p x}<0\left|T\left\{\bar{q} \sigma_{\mu \nu} q(x), \bar{q} \sigma_{\alpha \beta} q(0)\right\}\right| 0> \\
=\left(g_{\mu \alpha} g_{\nu \lambda}-g_{\mu \beta} g_{\nu \alpha}\right) \Pi_{1}\left(p^{2}\right) \\
+\left(g_{\mu \alpha} p_{\nu} p_{\beta}-g_{\nu \alpha} p_{\mu} p_{\beta}-g_{\mu \beta} p_{\nu} p_{\alpha}+g_{\nu \beta} p_{\nu} p_{\alpha}\right) \Pi_{2}\left(p^{2}\right) .
\end{array}
$$

The tensor susceptibility is determined by $\Pi_{1}(0)$.

We cannot determine the value of the polarization operator for $p^{2}=0$ directly but we can use the procedure which was suggested in [14] and consists in two steps: 1) Determination of parameters of a model for the spectral density of the correlator; 2) Evaluation of the correlator from a dispersion relation by using this model spectral density. This technique has been applied to find the value of magnetic susceptibility of quark condensate [14] and other induced quark condensates in the presence of an external vector field [7].

\subsection{QCD sum rule and spectral density}

Using OPE we found that

$$
\begin{gathered}
\Pi_{1}\left(Q^{2}\right)=\frac{1}{8 \pi^{2}} Q^{2} \ln \left(Q^{2} / \Lambda^{2}\right)+\frac{1}{24 Q^{2}}<\frac{\alpha_{s}}{\pi} G^{2}>_{0} \\
-\frac{4}{9}\left(\frac{1}{9}-\frac{1}{3}+\frac{2}{3}+1\right) \frac{\alpha_{s}}{\pi} \frac{\left(4 \pi^{2}<\bar{\psi} \psi>_{0}\right)^{2}}{4 \pi^{2} Q^{4}}+O\left(Q^{-6}\right) .
\end{gathered}
$$

Here the coefficients $\left(\frac{1}{9}-\frac{1}{3}+\frac{2}{3}+1\right)$ correspond to contributions of the operators: $\left(\bar{\psi} D_{\alpha} D_{\beta} D_{\gamma} \psi\right),\left(\bar{\psi} D_{\alpha} \psi G_{\beta \gamma}\right),\left(\bar{\psi} \psi D_{\alpha} G_{\beta \gamma}\right)$ and $(\bar{\psi} \psi)^{2}$.

The correlator of two tensor currents has been considered in [15]. Note that the coefficient for the last term in (2) disagrees with the result obtained in 15.

We can formally write the following dispersion relation for $\Pi_{1}\left(Q^{2}\right)$ :

$$
\Pi_{1}\left(Q^{2}\right)=\frac{1}{\pi} \int_{0}^{\infty} d s \frac{\operatorname{Im} \Pi_{1}(s)}{s+Q^{2}}
$$

This dispersion relation has ultraviolet divergence and can be presented in the form of dispersional relation with subtractions. It can be shown that 
the contribution of vector mesons with $J^{P C}=1^{--}$to the $\Pi_{1}\left(Q^{2}\right)$ is absent. Such mesons contribute to $\Pi_{2}\left(Q^{2}\right)$. The nonzero contribution into $\Pi_{1}\left(Q^{2}\right)$ comes from the hadronic states with $J^{P C}=1^{+-}$only. The lowest state with these quantum numbers is $B_{1}(1231)$ resonance.

Let us define

$$
<0\left|\bar{q} \sigma_{\mu \nu} q\right| B_{1}>=i f_{B} \varepsilon_{\mu \nu \rho \lambda} q_{\rho} e_{\lambda}
$$

Using the standard model for spectral density

$$
\frac{1}{\pi} \operatorname{Im} \Pi_{1}(s)=f_{B}^{2} m_{B}^{2} \delta\left(s-m_{B}^{2}\right)+\Theta\left(s-s_{0}\right) \frac{1}{\pi} \operatorname{Im} \Pi^{\text {pert. }}(s),
$$

we can find the following QCD sum rule for $f_{B}$ :

$$
\begin{array}{r}
\frac{M^{4}}{8 \pi^{2}}\left[1-e^{-\frac{s_{0}}{M^{2}}}\left(1+\frac{s_{0}}{M^{2}}\right)+\frac{\pi^{2}}{3} \frac{<\frac{\alpha_{s}}{\pi} G^{2}>_{0}}{M^{4}}-\frac{52}{81} \frac{\alpha_{s}}{\pi} \frac{2\left(4 \pi^{2}<\bar{\psi} \psi>_{0}\right)^{2}}{M^{6}}\right] \\
=f_{B}^{2} m_{B}^{2} e^{-\frac{m_{B}^{2}}{M^{2}}}
\end{array}
$$

Here $m_{B}=1.23 \mathrm{GeV}$ is the mass of $B_{1}$-meson, $s_{0}$ is the continuum threshold, $\frac{\alpha_{s}}{\pi} \simeq 0.1, \operatorname{Im} \Pi_{1}^{\text {pert. }}(s)$ is the imaginary part of the perturbative contribution, $<\frac{\alpha_{s}}{\pi} G^{2}>_{0}=0.012 G e V^{4}, 4 \pi^{2}\left|<\bar{\psi} \psi>_{0}\right|=0.55 G e V^{3}$.

The best fit for QCD sum rule (6) gives: $8 \pi^{2} f_{B}^{2}=2.4-2.6 G e V^{2}$ with $s_{0} \simeq 3 \mathrm{GeV}^{2}$. Thus, we have determined the parameters of the model spectral density (5).

\subsection{Dispersion Relation}

The tensor susceptibility is defined by the value of $\Pi_{1}\left(Q^{2}\right)$ for $Q^{2}=0$. As noted in the previous section, the dispersional relation (3) has ultraviolet divergence. The nature of this divergence is clear: the perturbative quark propagator in the presence of an external field cannot be expanded near the point $x^{2}=0$. On other hand, the perturbative part of this propagator is considered separately in the expression for quark propagator in the constant external tensor field (see eq.(11) [9]). So, to avoid double counting, we have to subtract the perturbative contribution from $\Pi_{1}\left(Q^{2}\right)$. This procedure gives 


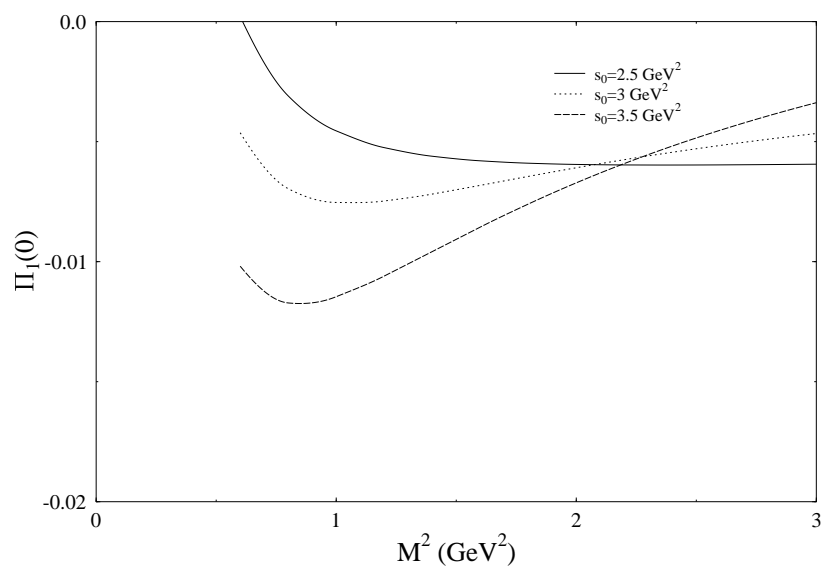

Figure 1:

the following result for the nonperturbative part of polarization operator $\Pi^{n p .}$ :

$$
\Pi_{1}^{n p .}(Q)=\frac{1}{\pi} \int_{0}^{\infty} \frac{\operatorname{Im} \Pi_{1}(s)-\operatorname{Im} \Pi_{1}^{\text {pert. }}(s)}{s+Q^{2}} d s .
$$

For the value $\Pi_{1}^{n p}$. we can use a dispersion relation without subtractions. This procedure has been suggested in [7] and gives a finite and selfconsistent result.

In the case of tensor susceptibility the value for $\Pi_{1}^{n p .}(0)$ is

$$
\Pi_{1}^{n p \cdot}(0)=f_{B}^{2}-\frac{s_{0}}{8 \pi^{2}}
$$

Using the QCD sum rule for $f_{B}$ (6) we can evaluate $\Pi_{1}^{n p .}(0)$. The dependence of this parameter of the nonperturbative propagator on $M^{2}$ for different $s_{0}$ is depicted in Fig.1.

From Fig.1 it is clear that

$$
\Pi_{1}^{n p \cdot}(0) \simeq-0.008 G e V^{2} .
$$

The previous estimations of [8, 9] are:

$$
\frac{1}{12} \Pi(0)=\Pi_{1}^{n p .} \simeq 0.002 G e V^{2} .
$$


Notice, that the result of present paper has opposite sign and is several times larger than the privious estimations [8, 9].

\section{Discussion}

The result obtained in this paper contradicts the prediction which has been made in [8, 9]. To understand what is a reason of such disagreement, let us consider the dispersion relation for the polarization operator

$$
\Pi\left(Q^{2}\right)=i \int d^{4} x e^{i q x}<0\left|T\left\{\bar{q} \sigma_{\mu \nu} q(x), \bar{q} \sigma_{\mu \nu} q(0)\right\}\right| 0>
$$

which has been used in [8, 9]. From eq.(1) it is clear that

$$
\Pi(0)=12 \Pi_{1}(0) .
$$

In Ref. [8] is was pointed that the perturbative contribution to correlator

(11) is absent. It means that the dispersional relation for $\Pi\left(Q^{2}\right)$ gives a finite result for $\Pi(0)$ and we do not need to subtract the perturbative contribution. It was suggested to use $\rho\left(1^{--}\right)$and $B_{1}\left(1^{+-}\right)$dominance to estimate $\Pi(0)$. It means that the dispersion relation for $\Pi(0)$ has the following form:

$$
\Pi(0)=\frac{1}{\pi} \int \frac{\rho_{\rho}(s)+\rho_{B}(s)}{s} d s
$$

where only the contributions of $\rho$ - and $B_{1}$-mesons are taken into accpount. Perturbative contribution to the sum $\rho_{\rho}(s)+\rho_{B}(s)$ is absent. Naively we can expect that the meson dominance model can give a reasonable prediction for $\Pi(0)$. However, our results $(9, \sqrt[10]{10})$ indicate that something is wrong.

To understand the source of discrepancy between two ways of the determinations of the correlator, let us write the following models for these spectral densities:

$$
\begin{array}{r}
\rho_{\rho}(s)=c_{\rho} \delta\left(s-m_{\rho}^{2}\right)+\Theta\left(s-s_{\rho}\right) \rho^{\text {pert. }}(s) \\
\rho_{B}(s)=c_{B} \delta\left(s-m_{B_{1}}^{2}\right)-\Theta\left(s-s_{B}\right) \rho^{\text {pert. }}(s) .
\end{array}
$$

Here we use the fact that the perturbative contribution in the two channels is equal to each other and has opposite sign. It is important to note that it is possible to separate the contribution $\rho$ - and $B$-meson channels starting from 
correlator (11). The spectral densities of these two channels have nonvanishing perturbative contribution and they are taking into account in (14,15).

Note that the thresholds $s_{\rho}$ and $s_{B}$ are not equal to each other. It means, that there is additional (to $\rho$ and $B$-meson) contribution in dispersion relation (13)

$$
\int_{s_{\rho}}^{s_{B}} \frac{\rho^{\text {pert. }}(s)}{s} d s
$$

which was not taken into consideration in [8, 9] and it is a source of discrepancy between these results. It is possible to reproduce our result by taking into account this term.

Here we have presented the procedure which has to be used for correct evaluation of induced condensates. Naive ways of determination of these condensates may lead to wrong results. The case of tensor susceptibility is the good example demonstrating the importance of the procedure which is applied for calculation of such condensates.

Finally we present our results for nucleon tensor charge which can be obtained from QCD sum rules in ref.[9] using our result for the tensor susceptibility:

$$
\begin{array}{r}
g_{T}^{u}=0.7 \pm 0.3 \\
g_{T}^{d} \simeq 0.01 .
\end{array}
$$

These results were obtained from QCD sum rules for $W_{3}$ (for $u$-tensor charge) and for $W_{1}$ (for $d$-tensor charge). The previous results are: $g_{T}^{u} \simeq 1.33 \pm 0.53$ and $g_{T}^{d} \simeq 0.04 \pm 0.02$. The tensor charge of nucleon is related to the first moment of the transversely distribution function $h_{1}(x)$. It can be measured in the Drell-Yan process with both beam and target transversely polarized.

\section{Acknowledgements:}

We are very thankful to B.L. Ioffe for fruitful discussions and to R. Peschanski for important remarks. This work was supported in part by INTAS Grant 93-0283 and by Swiss National Science Foundation. 


\section{References}

[1] M.A. Shifman, A.I. Vainshtein and V.I. Zakharov, Nucl. Phys. B147 (1979) 385, 448.

[2] M.A. Shifman, (ed.), "Vacuum Structure and QCD Sum Rules", Amsterdam, Netherlands: North-Holland (1992).

[3] B.L. Ioffe, Nucl. Phys. B188 (1981) 317.

[4] B.L. Ioffe and A.V. Smilga, JETP Lett. 37 (1983) 298; Nucl. Phys. B232 (1984) 109.

[5] Ya.Ya. Balitskii and A.V. Yung, Phys. Lett. B129 (1983) 328.

[6] V.M. Belyaev and Ya.I. Kogan, JETP Lett. 37 (1983) 730.

[7] V.M. Belyaev and I.I. Kogan Int.J.Mod.Phys. A8 (1993) 153; preprint ITEP-12 (1984).

[8] Hanxim He and Xiangdong Ji, Phys. rev D52 (1995) 2960.

[9] Hanxim He and Xiangdong Ji, Phys. Rev. D54 (1996) 6897.

[10] R.L. Jaffe and X. Ji, Phys. Rev. Lett. 67 (1991) 552; Nucl. Phys. B375 (1992) 527.

[11] J. Ralston and D.E. Soper, Nucl. Phys. B152 (1979) 109.

[12] B.L. Ioffe and A. Khodjamirian, Phys. Rev. D51 (1995) 3380.

[13] B.l. Ioffe, JETP Lett. 42 (1985) 327; V.M. Belyaev and B.L. Ioffe, Nucl. Phys. B310 (1988) 548.

[14] V.M. Belyaev and Ya.I. Kogan, Yad. Fiz. 40 (1984) 1035.

[15] J. Govaerts, L.J. Reinders, F. De Viron and J. Weyers, Nucl.Phys. B283 (1987) 706. 